\title{
Correlates Between Force and Postural Tremor in Older Individuals with Essential Tremor
}

\author{
Justin J. Kavanagh ${ }^{1}$ • Justin W.L. Keogh ${ }^{2,3,4}$
}

(C) Springer Science+Business Media New York 2015

\begin{abstract}
Essential tremor (ET) is commonly associated with kinetic tremor. However, other forms of tremor, such as force and postural tremor, may occur in ET with less severity. This study objectively assessed force and postural tremor characteristics in ET with the purpose of identifying the relationships between these tremors. Ten individuals with ET (age $71 \pm$ 5 years) and ten healthy controls (age $70 \pm 5$ years) participated in the study. Force tremor was quantified as fluctuations in index finger abduction force during isometric contractions at $10 \%$ maximum voluntary contraction (MVC) and $60 \%$ MVC. Postural tremor was quantified as index finger acceleration when the subjects held their entire arm unsupported, and when their arm was supported so that only the index finger could move. Time- and frequency-domain parameters were extracted from tremor data, and then correlations within, and between, tremor subtypes were examined. ET force tremor was dependent on contraction intensity whereas postural tremor was unaffected by the level of limb support. Significant correlations existed between frequency components of postural tremor and force tremor amplitude. Force tremor amplitude normalised to the level of contraction intensity correlated to the proportion of power for postural tremor. These correlations
\end{abstract}

Justin J. Kavanagh

j.kavanagh@griffith.edu.au

1 Centre for Musculoskeletal Research, Griffith University, Gold Coast, QLD 4222, Australia

2 Faculty of Health Sciences and Medicine, Bond University, Gold Coast, Australia

3 Human Potential Centre, AUT University, Auckland, New Zealand

4 Cluster for Health Improvement, Faculty of Science, Health, Education and Engineering, University of the Sunshine Coast, Sippy Downs, Australia were observed for both contraction intensities and both levels of postural support. The proportion of power represents the output of central oscillators in ET patients and therefore correlated well to force tremor. Given that significant relationships existed between spectral features of postural tremor and the overall force tremor amplitude, it is clear that these tremor modalities are not completely independent in older adults with ET.

Keywords Force tremor · Postural tremor · Frequency analysis $\cdot$ Upper limb $\cdot$ Isometric contraction

\section{Introduction}

Essential tremor (ET) is the most common pathologic tremor in older adults, affecting up to 1 in 25 individuals over 45 years [1], and as many as 1 in 16 individuals over 65 years $[2,3]$. Although the aetiology of ET is still unclear, a body of evidence is developing that implicates neuroanatomical changes in the cerebellum which causes movement dysfunction via cerebello-thalamo-cortical pathways [4-6]. The exacerbated tremor that is observed with ET has long been suggested to reflect enhanced motor unit synchronisation [7-9], which has only recently been confirmed via direct examination of motor unit activity [10].

Clinical diagnosis of pathologies often relies on visual rating scales to separate different forms of tremors. For example, the hallmark of ET is kinetic tremor, which tends to be most prevalent for movements about the wrist joint [11-13]. However, other forms of tremor, such as force and postural tremor, may exist in a less severe capacity in individuals with ET [11]. Force tremor is typically associated with muscle contraction, where tasks such as gripping or carrying objects can cause exacerbated tremor. Postural tremor, on the other hand, 
is largely associated with oscillations that arise from reflex activity and emerges when a limb is held against gravity. Clearly, a common feature of the tasks that produce force and postural tremor is that voluntary muscle contractions must be performed. Although the strength of contraction may differ-i.e. low magnitudes for postural tasks $[14,15]$ and potentially higher magnitudes for force generating tasks $[16$, 17] - there is still a need to regulate muscle activity to achieve a steady limb position.

Given that muscle activity underlies each form of tremor, and enhanced motor unit activity is typical in ET populations, it stands to reason that these tremors will be correlated and are not completely independent. Therefore, the purpose of this study was to examine two forms of tremor in older individuals with ET. Force tremor was examined during a low-intensity and high-intensity isometric contraction to determine if the level of force generated influences characteristics of force tremor. Postural tremor was assessed when holding an entire limb against gravity, and also when the limb was supported, to determine if the degree of limb support influences characteristics of postural tremor. Time-domain and frequency-domain correlations within, and between, tremor subtypes were then examined. It was hypothesised that substantial differences would occur in force tremor characteristics for the two contraction intensities, and only minimal differences would emerge in postural tremor characteristics for the two postural positions. It was also hypothesised that time- and frequencydomain characteristics of postural tremor would significantly correlate with force tremor.

\section{Materials and Methods}

\section{Participants}

Ten individuals with essential tremor (age $71 \pm 5$ years, age range 61-77 years, height $174 \pm 8 \mathrm{~cm}$, weight $84 \pm$ $9 \mathrm{~kg}$, six male) and ten healthy controls $(70 \pm 5$ years, age range $60-76$ years, height $168 \pm 8 \mathrm{~cm}$, weight $74 \pm$ $8 \mathrm{~kg}$, four male) participated in the study. To be included, ET participants required a diagnosis of ET for at least 1 year that was confirmed by a neurologist. ET participants were excluded if they had a diagnosis of head tremor without upper limb tremor, or were required to alter their medication 1 month leading up to the study. Individuals who had osteoarthritis, a recent history ( $<3$ years) of upper limb musculoskeletal injury, or any history of upper limb surgery were excluded. The revised Washington Heights-Inwood Genetic Study of Essential Tremor (WHIGET) tremor rating scale was used to indicate tremor severity of subjects during screening (rating of $1, n=4$; rating of $2, n=1$; rating of $3, n=4$; rating of $4, n=$ 1). All procedures were approved by the Institutional
Human Research Ethic Committee and written informed consent was obtained prior to subject participation.

\section{Tremor Assessment}

Force tremor was quantified as fluctuations in index finger abduction force when the subject's performed isometric contractions at $10 \%$ of maximum voluntary contraction (MVC) and $60 \%$ MVC. All subjects were seated and the palmar surface of their hand was placed flat on a custom-designed stainless steel device where the thumb was abducted and supported by a solid bracket, and the forearm and digits $3-5$ were secured [18]. Abduction force was measured at the proximal interphalangeal joint for the index fingers with an Ultra Precision Mini Load Cell (Transducer Techniques, MDB-5). Prior to experimental testing, MVC was determined using the same apparatus outlined above. To accommodate for the larger abduction forces, an Applied Measurements Xtrans load cell with a range of $250 \mathrm{~N}$ was used instead of the highsensitivity load cell.

Postural tremor was quantified as index finger acceleration when the subjects held their entire arm unsupported against gravity, and when their arm and hand was supported so that only the index finger was held against gravity. A lightweight NL261 uniaxial accelerometer (mass $2.55 \mathrm{~g}$, range $\pm 1.7 \mathrm{G}$, Digitimer Ltd.) was attached over the dorsal distal aspect of the index finger. The accelerometer was calibrated by zero balancing in DC mode on a level surface prior to testing in AC mode. For the unsupported condition, the seated subjects were required to extend their arm to $90^{\circ}$ with the torso and extend the index finger while forming a loose fist with the other digits. For the supported condition, the forearm and hand were secured to a table with Velcro while forming a loose fist, so that the principal movement against gravity was due to muscles spanning the metacarpophalangeal joint. Both force and acceleration data were sampled at $1000 \mathrm{~Hz}$ using customdesigned Spike2 software (Cambridge Electronic Design). Three 15-s trials were obtained for the more affected and less affected limb for all conditions.

\section{Data Analysis}

Finger abduction force was low-pass filtered with a fourthorder Butterworth filter set with a cut-off frequency of $40 \mathrm{~Hz}$. Absolute force tremor amplitude was quantified using the standard deviation (SD) of force. Given that tremor amplitude is influenced by the magnitude of force being generated, the coefficient of variation $(\mathrm{CV})$ of force was also calculated to provide a normalised measure of tremor. Finger acceleration data were also low-pass filtered with a fourth-order Butterworth filter set with a cut-off frequency of $40 \mathrm{~Hz}$. The amplitude of postural tremor was calculated as the root-meansquare (RMS) of the acceleration signal. All force and 
acceleration analyses were performed on the middle $8 \mathrm{~s}$ of data where steady state was achieved.

Force and acceleration data were examined in the frequency domain using Welch's averaged periodogram method (frequency resolution $0.130 \mathrm{~Hz}$ ). Force power spectra were examined in the $0-3-\mathrm{Hz}$ bandwidth for both the ET and control group to examine task-specific features of performing isometric contractions. Furthermore, force and acceleration data were examined in the 3-12-Hz bandwidth to capture the commonly reported $\sim 5-7-\mathrm{Hz}$ tremor associated with ET, and $8-12-\mathrm{Hz}$ tremor associated with healthy physiological tremor in the control group. Within these bandwidths, the frequency of peak power, and the proportion of the peak power compared to the power in the entire signal were calculated. All data analyses in the study were performed using Matlab (R2011b, Mathworks) software.

\section{Statistical Analysis}

One-way analysis of variance (ANOVA) was used to determine if between-limb differences existed for the time and frequency domain variables extracted from the force tremor and postural tremor data. If no statistical differences emerged between limbs, data would be pooled and one-way ANOVAs used to determine if differences existed for force tremor characteristics when performing the $10 \% \mathrm{MVC}$ and $60 \% \mathrm{MVC}$ conditions, and if power spectral characteristic differed between the 0-2- and 3-12-Hz bandwidth. One-way ANOVA was also used to determine if differences existed for postural tremor characteristics when performing the supported and unsupported limb conditions. Linear regression analysis was applied to global measures of tremor amplitude obtained during the force tasks (SD of force and CV of force) as well as during the postural tasks (RMS acceleration). Spectral parameters were also explored using linear and multiple linear regressions to identify potential mechanisms underlying changes in the global measures of tremor. All statistical analysis was performed using IBM SPSS Statistics (version 21) and the significance level was set at 0.05 .

\section{Results}

\section{Force Tremor and Postural Tremor Characteristics}

Even though each ET subject indicated that they had a more affected limb and a less affected limb, no significant betweenlimb differences were identified for any force tremor (Table 1) or postural tremor variable (Table 2). Similarly, there were no between-limb differences identified for the healthy control group.

Several significant differences were identified for force tremor when subjects performed isometric contractions at
$10 \%$ MVC and $60 \%$ MVC (Fig. 1). For the ET group, higher SD of force was identified for the $60 \%$ MVC condition compared to that for the $10 \% \mathrm{MVC}$ condition $(0.421 \pm 0.223 \mathrm{vs}$ $0.149 \pm 0.080 \mathrm{~N}, F(1,38)=23.53, p<0.001$, Table 1$)$, whereas lower $\mathrm{CV}$ of force was identified for the $60 \% \mathrm{MVC}$ condition compared to the $10 \%$ MVC condition $(4.19 \pm 2.08$ vs $8.03 \pm$ $4.97 \%, F(1,38)=9.74, p=0.003)$. For the control group, only the SD of force differed between contraction intensities, where a higher SD of force was identified for the $60 \% \mathrm{MVC}$ condition compared to that for the $10 \%$ MVC condition $(0.234 \pm$ 0.148 vs $0.069 \pm 0.026 \mathrm{~N}, F(1,38)=29.12, p<0.001)$.

Comparing spectral parameters between the two contraction intensities revealed different force tremor profiles in the ET group. In particular, for the ET group, the proportion of total signal power that the peak represented in each bandwidth was inversely related to the contraction intensity (this was not observed in the control group). That is, the proportion of power in $0-2-\mathrm{Hz}$ bandwidth was greater for the $60 \% \mathrm{MVC}$ con-

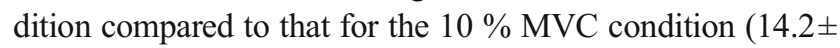
2.5 vs $11.5 \pm 4.8 \%, F(1,38)=4.92, p=0.032)$, whereas the proportion of power in $3-12-\mathrm{Hz}$ bandwidth was greater for the $10 \%$ MVC condition compared to that for the $60 \%$ MVC condition $(4.8 \pm 4.7$ vs $1.6 \pm 1.3 \%, F(1,38)=7.30, p=$ 0.010 , Table 1). In regards to the proportion of power in each bandwidth, both groups had greater power in the lower frequency bandwidth than in the higher frequency bandwidth. The ET group had significantly greater proportion of power in the $0-2-\mathrm{Hz}$ band than in the $3-12-\mathrm{Hz}$ band for the $10 \%$ MVC $(11.5 \pm 4.9$ vs $4.8 \pm 4.7, F(1,38)=12.30, p<0.001)$ and $60 \%$ MVC conditions $(14.2 \pm 2.5$ vs $1.6 \pm 1.3 \%, F(1,38)=$ $13.41, p<0.001$ ), which was consistent with the control group $10 \%$ MVC $(19.8 \pm 14.7$ vs $0.6 \pm 0.4 \%, F(1,38)=15.76$, $p<0.001)$ and $60 \% \mathrm{MVC}(16.3 \pm 10.1$ vs $0.5 \pm 0.4 \%, F(1$, $38)=12.89, p<0.001)$. Unlike force tremor, the different postural tremor conditions did not alter tremor characteristics of individuals with ET or the healthy control group (Table 2).

\section{Relationships Between Force Tremor Assessments}

Positive correlations emerged for the global measures of force tremor and postural tremor. Significant correlations existed in the ET group between the $10 \% \mathrm{MVC}$ and the $60 \% \mathrm{MVC}$ condition for SD of force $\left(r^{2}=0.642, p<0.001\right)$, as well as for the CV of force $\left(r^{2}=0.601, p<0.001\right.$, Fig. 2). These similarities between contraction intensities appear to be mediated by activity in the $3-12-\mathrm{Hz}$ spectral bandwidth, as significant correlations existed between the $10 \% \mathrm{MVC}$ and the $60 \% \mathrm{MVC}$ condition for the peak frequency of force $\left(r^{2}=0.390, p=\right.$ 0.003 ), and the proportion of total signal power that the peak represented $\left(r^{2}=0.361, p=0.020\right)$. In contrast to the ET group, the control group exhibited weaker correlations between the same force variables. Of note were the significant correlations 
Table 1 Force tremor characteristics for older individuals with essential tremor and healthy older controls

\begin{tabular}{|c|c|c|c|c|c|c|c|}
\hline & & \multirow[b]{2}{*}{$\mathrm{SD}(N)$} & \multirow[b]{2}{*}{$\mathrm{CV}(\%)$} & \multicolumn{2}{|c|}{$0-2-\mathrm{Hz}$ bandwidth } & \multicolumn{2}{|c|}{ 3-12-Hz bandwidth } \\
\hline & & & & $\begin{array}{l}\text { Peak frequency } \\
(\mathrm{Hz})\end{array}$ & $\begin{array}{l}\text { Proportional } \\
\text { power (\%) }\end{array}$ & $\begin{array}{l}\text { Peak frequency } \\
(\mathrm{Hz})\end{array}$ & $\begin{array}{l}\text { Proportional } \\
\text { power (\%) }\end{array}$ \\
\hline \multicolumn{8}{|l|}{ Essential tremor } \\
\hline \multirow[t]{2}{*}{$10 \% \mathrm{MVC}$ force tremor } & More affected & $0.153 \pm 0.090$ & $8.60 \pm 4.12$ & $0.54 \pm 0.20$ & $13.5 \pm 5.2$ & $5.30 \pm 0.96$ & $4.7 \pm 5.7$ \\
\hline & Less affected & $0.145 \pm 0.073$ & $7.46 \pm 5.87$ & $0.60 \pm 0.19$ & $9.4 \pm 3.5$ & $5.61 \pm 0.72$ & $4.9 \pm 4.6$ \\
\hline \multirow[t]{2}{*}{$60 \% \mathrm{MVC}$ force tremor } & More affected & $0.419 \pm 0.255$ & $4.32 \pm 2.31$ & $0.56 \pm 0.20$ & $14.0 \pm 2.6$ & $5.54 \pm 0.98$ & $1.4 \pm 1.5$ \\
\hline & Less affected & $0.422 \pm 0.200$ & $3.97 \pm 1.93$ & $0.61 \pm 0.24$ & $14.3 \pm 2.5$ & $5.69 \pm 0.83$ & $1.9 \pm 1.1$ \\
\hline \multicolumn{8}{|l|}{ Healthy older } \\
\hline \multirow[t]{2}{*}{$10 \% \mathrm{MVC}$ force tremor } & Dominant & $0.057 \pm 0.024$ & $3.14 \pm 1.41$ & $0.49 \pm 0.12$ & $23.0 \pm 22.8$ & $7.98 \pm 0.41$ & $0.7 \pm 0.5$ \\
\hline & Non-dominant & $0.081 \pm 0.030$ & $4.70 \pm 2.32$ & $0.51 \pm 0.11$ & $16.7 \pm 6.8$ & $7.91 \pm 0.45$ & $0.5 \pm 0.4$ \\
\hline \multirow[t]{2}{*}{$60 \% \mathrm{MVC}$ force tremor } & Dominant & $0.223 \pm 0.149$ & $2.29 \pm 1.51$ & $0.66 \pm 0.19$ & $16.8 \pm 8.5$ & $7.97 \pm 0.45$ & $0.3 \pm 0.1$ \\
\hline & Non-dominant & $0.247 \pm 0.149$ & $2.67 \pm 1.21$ & $0.61 \pm 0.23$ & $15.9 \pm 12.8$ & $7.99 \pm 0.37$ & $0.6 \pm 0.5$ \\
\hline
\end{tabular}

$S D$ standard deviation, $C V$ coefficient of variation, $M V C$ maximum voluntary contraction

between the $10 \% \mathrm{MVC}$ and the $60 \% \mathrm{MVC}$ condition for SD of force $\left(r^{2}=0.242, p=0.028\right)$.

\section{Relationships Between Postural Tremor Assessments}

Similar to the global measures of force tremor, a significant correlation existed in the ET group between the supported and unsupported limb conditions for RMS acceleration $\left(r^{2}=0.836, p<0.001\right.$, Fig. 2). These similarities between support conditions were also reflected in spectral parameters, as significant correlations were identified between the supported and unsupported conditions for the peak frequency of acceleration $\left(r^{2}=0.566, p<0.001\right)$, and the proportion of total signal power that the peak represented $\left(r^{2}=0.530, p=0.001\right)$. Once again, the control group exhibited weaker correlations between the same variables. Of note were the significant correlations between the supported and unsupported limb conditions for RMS acceleration $\left(r^{2}=0.572, p<0.001\right)$.

\section{Relationships Between Postural and Force Tremor}

Postural tremor measures did not significantly correlate with the absolute measures of force tremor, i.e. the SD of force for $10 \%$ MVC or $60 \%$ MVC. However, significant correlations were consistently identified in the ET group for the proportion of power the peak frequency represented in postural tremor and the normalised measures of force tremor, i.e. the $\mathrm{CV}$ of force for $10 \%$ MVC or $60 \%$ MVC. Significant correlations existed between the $\mathrm{CV}$ of force for the $10 \% \mathrm{MVC}$ condition and the proportion of power for the supported limb condition $\left(r^{2}=0.432, p=0.002\right.$, Fig. 3$)$, as well as the proportion of power for the unsupported limb condition $\left(r^{2}=0.476, p<0.001\right)$. When the proportion of power for the supported and

Table 2 Postural tremor characteristics for older individuals with essential tremor and healthy older controls

\begin{tabular}{|c|c|c|c|c|}
\hline & & $\begin{array}{l}\text { RMS amplitude } \\
\text { (G) }\end{array}$ & $\begin{array}{l}\text { Peak frequency } \\
(\mathrm{Hz})\end{array}$ & $\begin{array}{l}\text { Proportional } \\
\text { power }(\%)\end{array}$ \\
\hline \multicolumn{5}{|l|}{ Essential tremor } \\
\hline \multirow[t]{2}{*}{ Unsupported postural tremor } & More affected & $0.095 \pm 0.096$ & $6.78 \pm 0.81$ & $11.0 \pm 7.4$ \\
\hline & Less affected & $0.064 \pm 0.033$ & $6.84 \pm 0.87$ & $9.9 \pm 6.8$ \\
\hline \multirow[t]{2}{*}{ Supported postural tremor } & More affected & $0.110 \pm 0.124$ & $6.32 \pm 1.15$ & $10.3 \pm 6.5$ \\
\hline & Less affected & $0.069 \pm 0.033$ & $6.34 \pm 0.86$ & $8.7 \pm 5.0$ \\
\hline \multicolumn{5}{|l|}{ Healthy older } \\
\hline \multirow[t]{2}{*}{ Unsupported postural tremor } & Dominant & $0.028 \pm 0.008$ & $8.01 \pm 0.57$ & $3.1 \pm 1.7$ \\
\hline & Non-dominant & $0.026 \pm 0.006$ & $8.21 \pm 0.53$ & $2.8 \pm 0.6$ \\
\hline \multirow[t]{2}{*}{ Supported postural tremor } & Dominant & $0.025 \pm 0.011$ & $8.41 \pm 0.46$ & $2.7 \pm 0.7$ \\
\hline & Non-dominant & $0.027 \pm 0.009$ & $8.37 \pm 0.53$ & $2.2 \pm 0.8$ \\
\hline
\end{tabular}

$R M S$ root-mean-square 

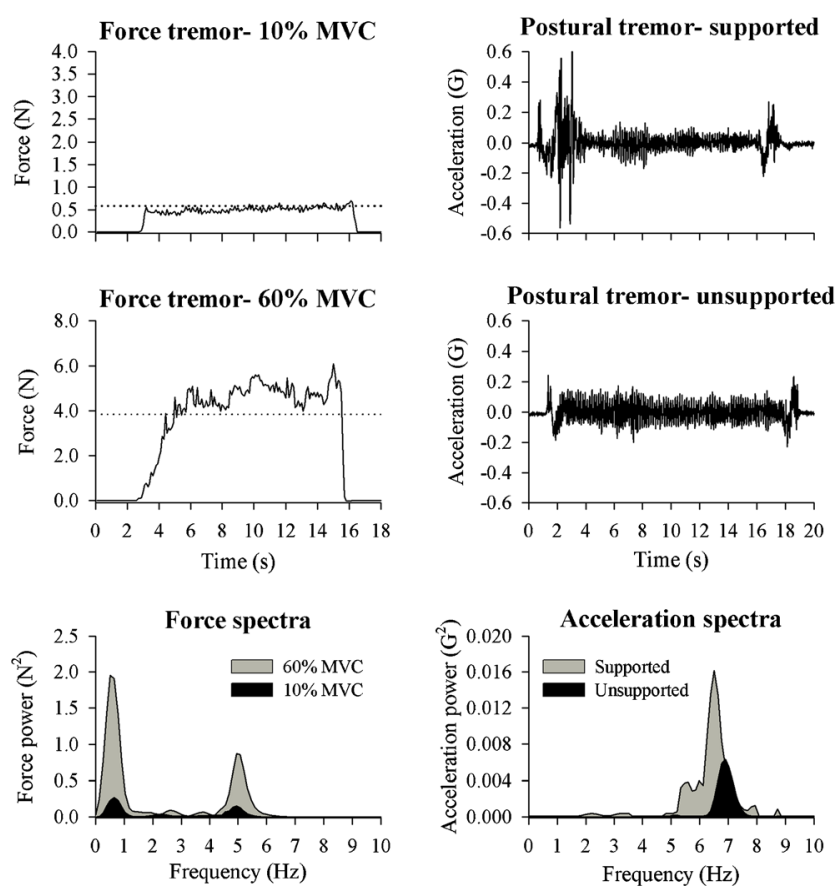

Fig. 1 Representative data illustrating raw force outputs for the $10 \%$ MVC and $60 \%$ MVC isometric contractions, and raw acceleration outputs for the supported and unsupported limb conditions. Power spectral plots for the force and acceleration data are presented in the lower plots. All data is for the more affected limb of a 70-year-old male with essential tremor

unsupported conditions was entered into a multiple linear regression, the predictive capability of postural tremor data improved slightly $\left(r^{2}=0.523, p=0.001\right)$.

Significant correlations also existed between the $\mathrm{CV}$ of force for the $60 \% \mathrm{MVC}$ condition and the proportion of power for the supported limb condition $\left(r^{2}=0.641\right.$, $p<0.001$ ), as well as the proportion of power for the unsupported limb condition $\left(r^{2}=0.544, p<0.001\right)$. When the proportion of power for the supported and unsupported conditions was entered into a multiple linear regression, the predictive capability of postural tremor data was once again minimally affected $\left(r^{2}=0.687, p<0.001\right)$. For the control group, a significant correlation was only revealed between the $\mathrm{CV}$ of force for the $10 \% \mathrm{MVC}$ condition and the proportion of power for the supported limb condition $\left(r^{2}=0.298, p=0.015\right)$.

\section{Discussion}

The purpose of this study was to examine two forms of tremor in older individuals with ET. Overall, ET force tremor was dependent on contraction intensity whereas postural tremor was unaffected by the level of limb support. Significant correlations existed between power spectral features of postural tremor and the overall force tremor amplitude, which indicates that these tremors were not independent of each other. With the current data set, no significant correlations existed between tremor metrics and age or duration of ET, which in part reflects the somewhat idiopathic and variable nature of the condition.

In alignment with our hypotheses, force tremor characteristics were different when ET patients performed low- and high-intensity steady state contractions. The absolute amplitude of finger abduction tremor was greatest for the highest contraction intensity, which agrees with previous studies of wrist extensor force generation in ET patients [17]. However, this absolute measure of force tremor is scaled to the level of overall contraction [19], which may mask changes in central nervous system (CNS) dynamics that are independent of contraction intensity. Our normalised measures of force tremor (CV of force) revealed that ET force tremor was actually greater for the $10 \% \mathrm{MVC}$ condition, which aligns with non-pathologic young and older adults $[19,20]$ and suggests that the CNS may be challenged when performing more refined tasks which do not require forceful muscle contractions.

Spectral analysis confirmed that a $0-1-\mathrm{Hz}$ force tremor component existed for ET patients and was similarly noted in the healthy older group. This index finger abduction frequency reflects elements of common drive to the motorneuron pool of the first dorsal interosseus when performing isometric contractions [21] and was therefore greater for the higherintensity task. A prominent $5-6-\mathrm{Hz}$ oscillatory component was also present in the force signal, which reflects the ensemble of elements which combine to cause ET specific tremor. Given the relationship between the $0-1-\mathrm{Hz}$ peak and the 5-6$\mathrm{Hz}$ peak during different contraction intensities, the overall contribution of 5-6-Hz component appears to be more influential on contraction steadiness in the lower-intensity than in the higher-intensity task. Once again, this suggests that the control of low-intensity contractions may be particularly influenced by mechanisms that underlie ET.

Neuroimaging has revealed that a multitude of structural [22], functional [23], and metabolic [24] changes may occur in supraspinal centres with the development of ET. While the force tracking task used in this study requires cerebellar control of eye movements and gaze fixation (i.e. the fastigial oculomotor region $[25,26]$ ), of particular interest is how CNS pathways regulate descending motor signals that activate the muscle of interest. fMRI has identified increased blood oxygen leveldependent (BOLD) activity in the primary and supplementary motor areas of cortex in ET patients, which is coupled to decreased BOLD activity in the cerebellum during gripping [27]. This suggests that ET is characterised by a hyperactive cortex and a hypoactive cerebellum during force generating tasks [27]. Regulating constant force produced with fingers has been linked to the lateral cerebellum and projections to the dentate nucleus [28], and not surprisingly, the functional 
Fig. 2 Linear regressions between force tremor and postural tremor conditions. Data are presented for SD of force (10\% MVC vs $60 \% \mathrm{MVC}), \mathrm{CV}$ of force (10\% MVC vs $60 \% \mathrm{MVC}$ ), and RMS acceleration (supported and unsupported). Filled symbols represent the more affected limb in the ET group or the dominant limb of individuals in the control group. Open symbols represent the less affected limb in the ET group or the non-dominant limb of individuals in the control group
Essential Tremor
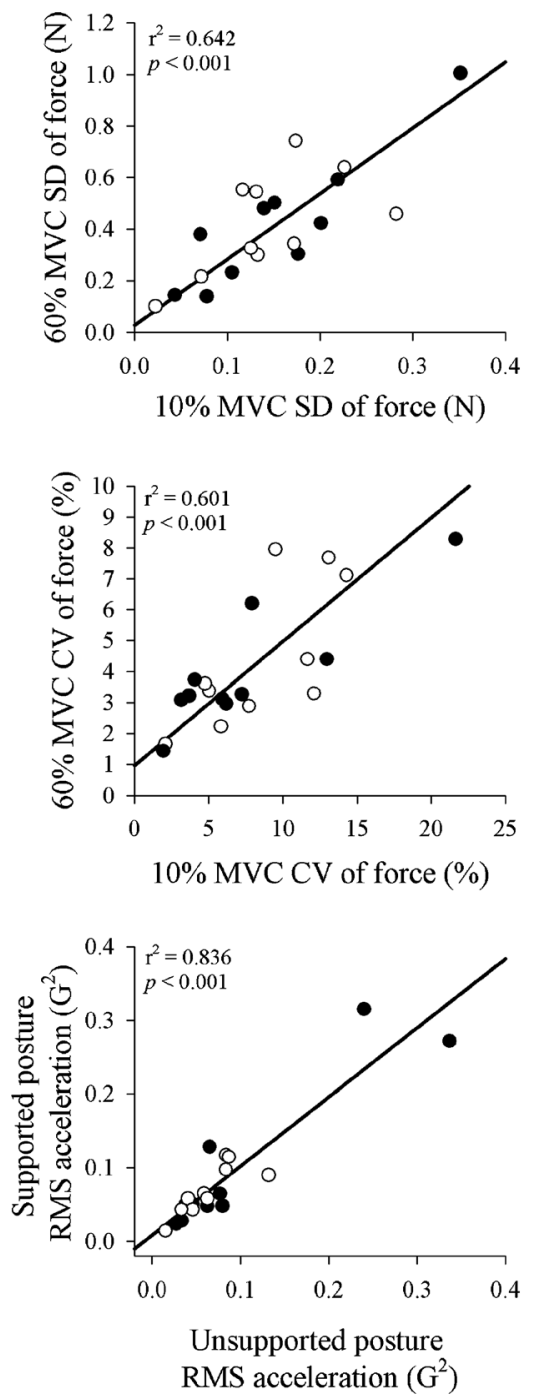

Healthy older
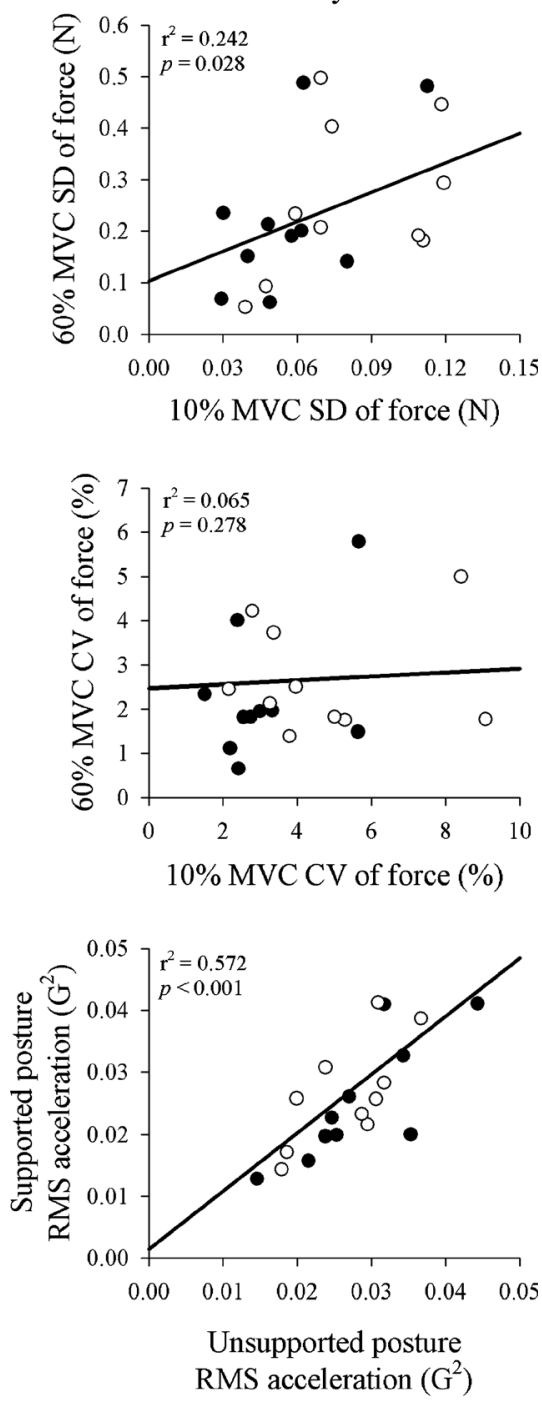

connectivity of cerebello-dento-thalamic tracts is compromised in ET patients to cause pronounced movement deficits of the arms and hands [29].

Postural tremor dynamics are largely influenced by the inertial characteristics of the limb segments(s) involved in the task, and therefore different inertial conditions may be created by changing the level of support available to the upper limb. Different levels of upper limb support did not yield significant postural tremor differences, which suggests that changing the inertial requirements of the task does not play an important role in regulating postural tremor in older individuals with ET. Given that postural tremor arises from a combination of central oscillators and peripheral reflexes [30,31], the results suggest that either (1) the mechanisms underlying the generation of postural tremor in older patients with ET is relatively unaffected by limb posture, or (2) the CNS of older ET patients is able to compensate for changes to one or more of these mechanisms to regulate postural tremor. Several studies have examined the effects of limb loading in ET patients, where applying a mechanical load to the hand is an effective inertial intervention that decreases postural tremor [32-34]. However, it should be noted that these studies examined extrinsic perturbations via limb loading, whereas the current study examined inertial effects by changing the number of limb segments which contribute to postural tremor.

A major finding in this study was that relationships were identified between force tremor and postural tremor. Tremor amplitude that was normalised to the amplitude of the isometric contraction was correlated to the proportion of power for postural tremor. This finding held true for both contraction intensities and both levels of postural support. Given that the proportion of power represents the culminated output of central oscillators, it is not surprising that normalised force tremor aligns with this postural tremor component. Fluctuations in limb position and steady state contraction ultimately arise from motor unit activity, and if neural inputs to the 
Fig. 3 Linear regressions between normalised force tremor and postural tremor conditions. Data are presented for $\mathrm{CV}$ of force (10\% MVC and $60 \% \mathrm{MVC}$ ) and the proportion of total signal power that the dominant frequency component represents in the acceleration signal (supported and unsupported limb conditions). Filled symbols represent the more affected limb in the ET group or the dominant limb of individuals in the control group. Open symbols represent the less affected limb in the ET group or the non-dominant limb of individuals in the control group

\section{Essential Tremor}
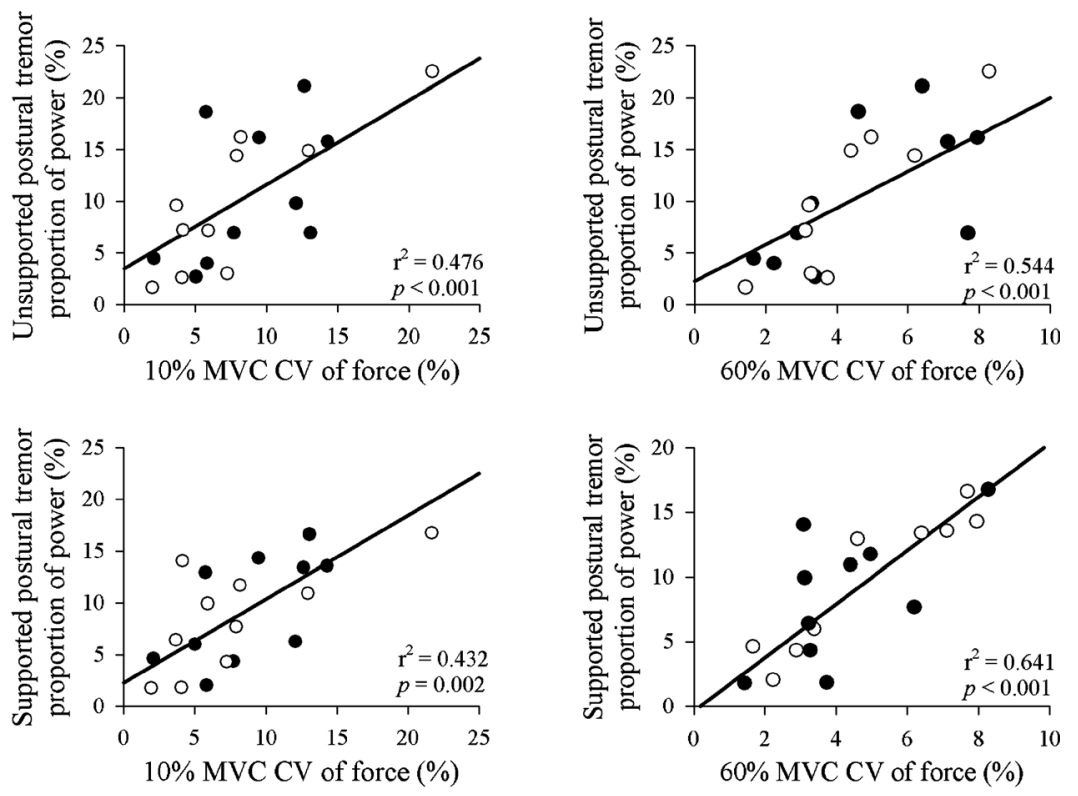

Healthy older
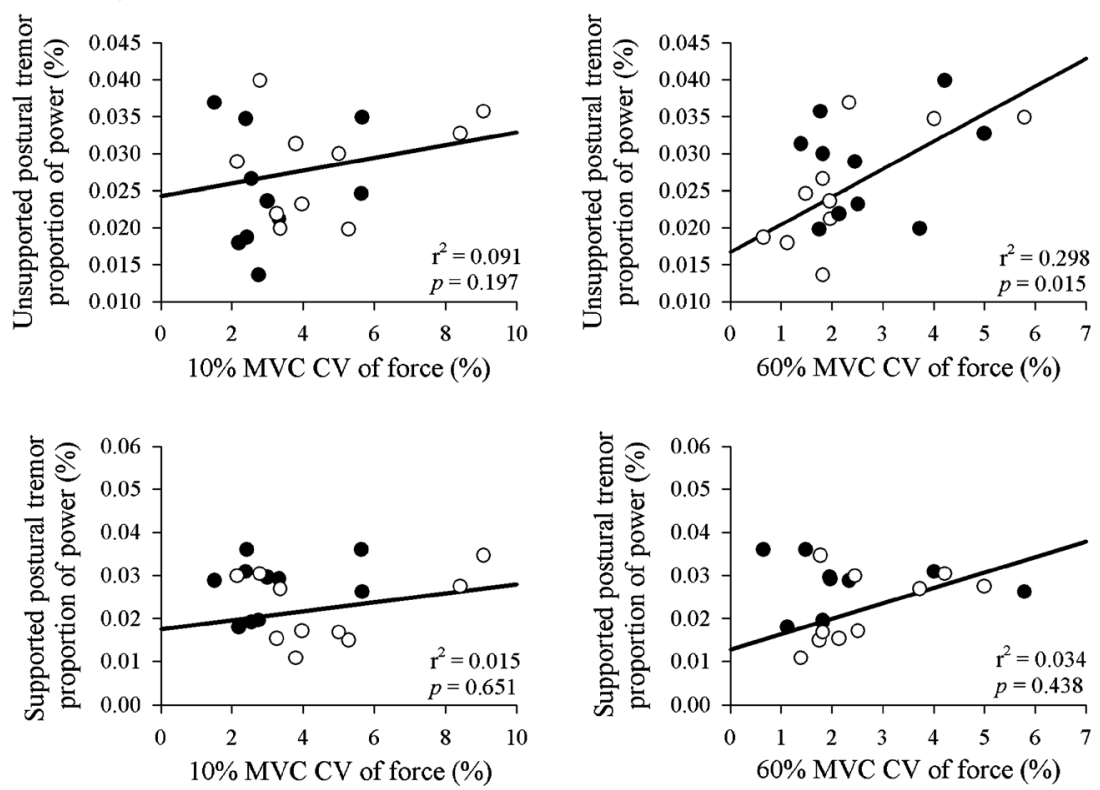

motorneuron pool have been affected due to ET dysfunction, then it might be expected that both postural and force tasks are compromised to some extent. Abnormal descending oscillatory signals in individuals with ET lead to greater motor unit entrainment in muscles involved with postural tremor and force tremor [17, 32]. Presumably, this enhanced entrainment plays a role in the correlations between tremor modalities.

Interestingly, if the goal of a tremor investigation is to examine time-domain characteristics of ET, there may not be benefits of performing variants of the same task. Regardless of whether SD or CV of force was examined, significant correlations still occurred with the low- and high-intensity contractions. Similarly, RMS acceleration during the supported and unsupported conditions was also significantly correlated. This has practical implications for the study of ET, as eliminating redundancy in experimental or clinical testing procedures may enhance the efficiency of working with this special population. It appears that the combination of normalised time-domain tremor, and the frequency content of the tremor signal, may provide the greatest insight to the dysfunction associated with ET. It is estimated that between 30 and $50 \%$ of ET diagnoses are incorrect $[11,35]$, so additional work on the defining features of ET is warranted. While it may be technical in nature, it is surprising that more assessments of 
clinical populations do not objectively examine time- and frequency-domain components of tremor, as the definition of tremor itself is an involuntary oscillatory movement of a body part.

Acknowledgments The authors wish to thank the International Essential Tremor Foundation for providing funds to undertake this study.

\section{Compliance with ethical standards}

Conflict of interest The International Essential Tremor Foundation provided a competitive research grant for this study to be performed.

The authors declare that there are no conflicts of interest.

All authors have read the manuscript and approve of submission. This paper has not been previously published and is not under consideration at another journal.

\section{References}

1. Dogu O, Sevim S, Camdeviren H, Sasmaz T, Bugdayci R, Aral M, et al. Prevalance of essential tremor: door-to-door neurologic exams in Mersin Province. Turkey Neurol. 2003;61:1804-6.

2. Louis ED, Ferreira JJ. How common is the most common adult movement disorder? Update on the worldwide prevalence of essential tremor. Mov Disord. 2010;25(5):534-41.

3. Louis ED, Marder K, Cote L, Wilder D, Tang MX, Lantigua R, et al. Prevalance of a history of shaking in persons 65 years of age and older: diagnostic and functional correlates. Mov Disord. 1996;11(1):63-9.

4. Louis ED, Lee M, Cortés E, Vonsattel JP, Faust PL. Matching asymmetry of tremor with asymmetry of postmortem cerebellar hemispheric changes in Essential Tremor. Cerebellum. 2014;13(4):462-70.

5. Helmich RC, Toni I, Deuschl G, Bloem BR. The pathophysiology of essential tremor and Parkinson's tremor. Curr Neurol Neurosci Rep. 2013;13(9):378.

6. Louis ED, Kuo SH, Vonsattel JP, Faust PL. Torpedo formation and Purkinje cell loss: modeling their relationship in cerebellar disease. Cerebellum. 2014;13(4):433-9.

7. Elek JM, Dengler R, Konstanzer A, Hesse S, Wolf W. Mechanical implications of paired motor unit discharges in pathological and voluntary tremor. Electroencephalogr Clin Neurophysiol. 1991;81(4):279-83.

8. Elble RJ, Deuschl G. An update on essential tremor. Curr Neurol Neurosci Rep. 2009;9(4):273-7.

9. McAuley JH, Marsden CD. Physiological and pathalogical tremors and rhythmic central motor control. Brain. 2000;123:1545-67.

10. Gallego JA, Dideriksen JL, Holobar A, Ibáñez J, Pons JL, Louis $\mathrm{ED}$, et al. Influence of common synaptic input to motor neurons on the neural drive to muscle in essential tremor. J Neurophysiol. 2015;113(1):182-91.

11. Louis ED. The primary type of tremor in essential tremor is kinetic rather than postural: cross-sectional observation of tremor phenomenology in 369 cases. Eur J Neurol. 2013;20(4):725-7.

12. Louis ED. Essential tremor. Lancet Neurol. 2005;4(2):100-10.

13. Brennan KC, Jurewicz EC, Ford B, Pullman SL, Louis ED. Is essential tremor predominantly a kinetic or a postural tremor? A clinical and electrophysiological study. Mov Disord. 2002;17(2):313-6.

14. Morrison S, Newell K. Limb stiffness and postural tremor in the arm. Mot Control. 2000;4:293-315.
15. Kavanagh JJ, Cresswell AG, Sabapathy S, Carroll TJ. Bilateral tremor responses to unilateral loading and fatiguing muscle contractions. J Neurophysiol. 2013;110(2):431-40.

16. Kavanagh JJ, Wedderburn-Bisshop J, Keogh JW. Resistance training reduces force tremor and improves manual dexterity in older individuals with Essential Tremor. J Mot Behav. 2015; In press.

17. Heroux ME, Pari G, Norman KE. The effect of contraction intensity on force fluctuations and motor unit entrainment in individuals with essential tremor. Clin Neurophysiol. 2010;121:233-9.

18. Kenway LC, Bisset LM, Kavanagh JJ. The effect of isometric contraction on the regulation of force tremor in the contralateral limb. Neurosci Lett. 2014;558:126-31.

19. Enoka RM, Christou EA, Hunter SK, Kornatz KW, Semmler JG, Taylor AM, et al. Mechanisms that contribute to differences in motor performance between young and old adults. J Electromyogr Kinesiol. 2003;13:1-12.

20. Burnett RA, Laidlaw DH, Enoka RM. Coactivation of the antagonist muscle does not covary with steadiness in old adults. J Appl Physiol. 2000;89(1):61-71.

21. Negro F, Holobar A, Farina D. Fluctuations in isometric muscle force can be described by one linear projection of low frequency components of motor unit discharge rates. J Physiol. 2009;15(587): 5925-38.

22. Benito-León J, Alvarez-Linera J, Hernández-Tamames JA, AlonsoNavarro H, Jiménez-Jiménez FJ, Louis ED. Brain structural changes in essential tremor: voxel-based morphometry at 3-Tesla. J Neurolog Sci. 2009;15(287):138-42.

23. Bucher SF, Seelos KC, Dodel RC, Reiser M, Oertel WH. Activation mapping in essential tremor with functional magnetic resonance imaging. Ann Neurol. 1997;41(1):32-40.

24. Louis ED, Shungu DC, Mao X, Chan S, Jurewicz EC. Cerebellar metabolic symmetry in essential tremor studied with $1 \mathrm{H}$ magnetic resonance spectroscopic imaging: implications for disease pathology. Mov Disord. 2004;19(6):672-7.

25. Pélisson D, Goffart L, Guillaume A. Control of saccadic eye movements and combined eye/head gaze shifts by the medio-posterior cerebellum. Prog Brain Res. 2003;142:69-89.

26. Robinson FR, Fuchs AF. The role of the cerebellum in voluntary eye movements. Annu Rev Neurosci. 2001;24:981-1004.

27. Neely KA, Kurani AS, Shukla P, Planetta PJ, Wagle Shukla A, Goldman JG, et al. Functional brain activity relates to 0-3 and 3-8 $\mathrm{Hz}$ force oscillations in Essential Tremor. Cerebral Cort. 2014; In press.

28. Vaillancourt DE, Thulborn KR, Corcos DM. Neural basis for the processes that underlie visually guided and internally guided force control in humans. J Neurophysiol. 2003;90(5):3330-40.

29. Buijink AWG, van der Stouwe AMM, Broersma M, Sharifi S, Groot PFC, Speelman JD, et al. Motor network disruption in essential tremor: a functional and effective connectivity study. Brain. 2015; In press.

30. Elble RJ, Randall JE. Mechanistic components of normal hand tremor. Electroencephalogr Clin Neurophysiol. 1978;44:72-82.

31. Fox JR, Randall JE. Relationship between forearm tremor and the biceps electromyogram. J Appl Physiol. 1970;29:103-8.

32. Heroux ME, Pari G, Norman KE. The effect of inertial loading of wrist postural tremor in essential tremor. Clin Neurophysiol. 2009;120:1020-9.

33. Bilodeau M, Keen DA, Sweeney PJ, Shields RW, Enoka RM. Strength training can improve steadiness in persons with essential tremor. Muscle Nerve. 2000;23(5):771-8.

34. Elble RJ. Physiologic and essential tremor. Neurology. 1986;36(2): 225-31.

35. Jain S, Lo SE, Louis ED. Common misdiagnosis of a common neurological disorder: how are we misdiagnosing essential tremor? Arch Neurol. 2006;63:1100-4. 


\section{Correlates Between Force and Postural Tremor in Older Individuals with Essential Tremor}

\section{Justin J. Kavanagh \& Justin W.L. Keogh}

The Cerebellum

ISSN 1473-4222

Cerebellum

DOI 10.1007/s12311-015-0732-2

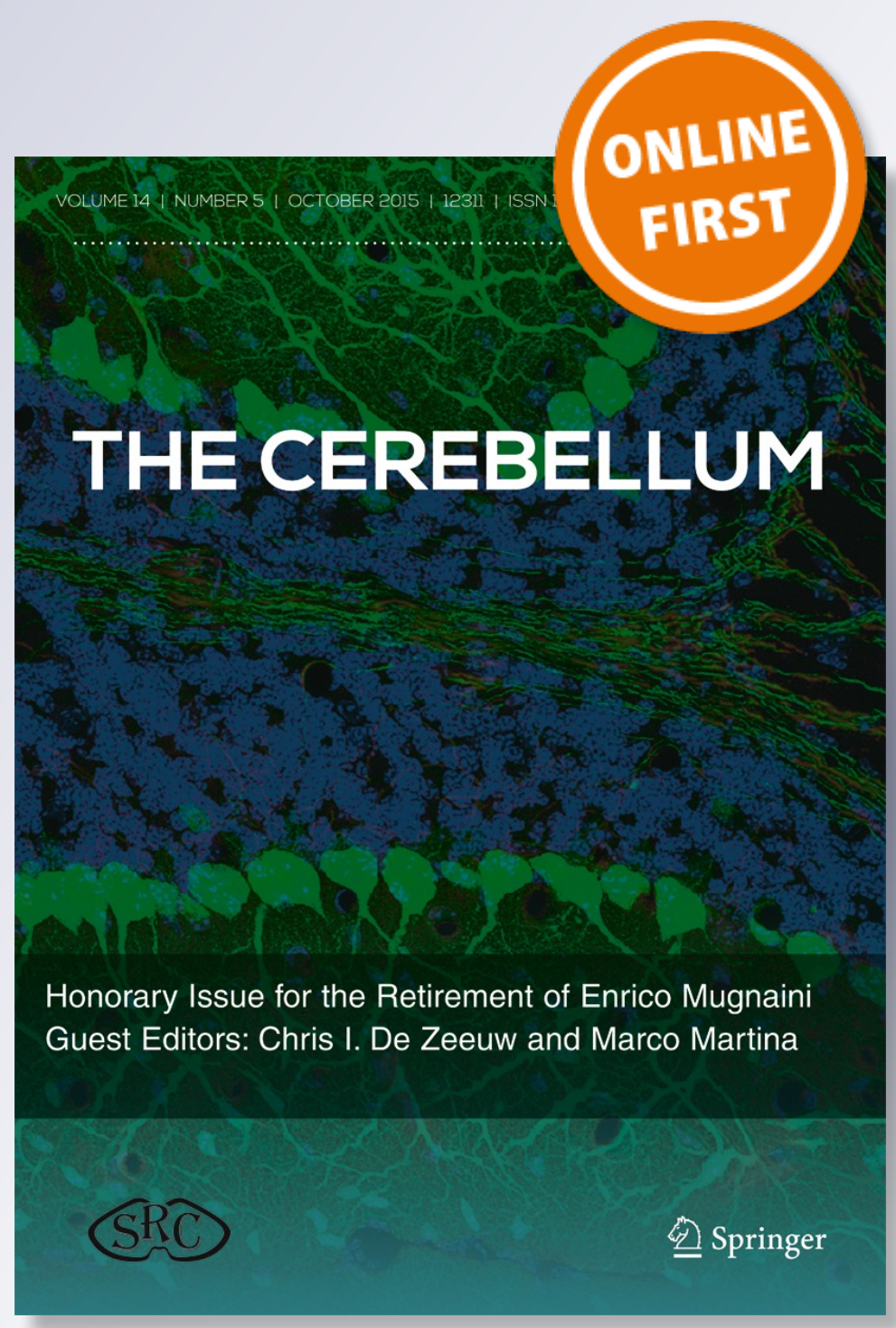

算 Springer 
Your article is protected by copyright and all rights are held exclusively by Springer Science +Business Media New York. This e-offprint is for personal use only and shall not be selfarchived in electronic repositories. If you wish to self-archive your article, please use the accepted manuscript version for posting on your own website. You may further deposit the accepted manuscript version in any repository, provided it is only made publicly available 12 months after official publication or later and provided acknowledgement is given to the original source of publication and a link is inserted to the published article on Springer's website. The link must be accompanied by the following text: "The final publication is available at link.springer.com". 